\title{
Burden of disease and clinical targets in adult patients with X-linked hypophosphatemia. A comprehensive review
}

\author{
S. Giannini ${ }^{1}(\mathbb{D}) \cdot$ M.L. Bianchi $^{2} \cdot$ D. Rendina ${ }^{3} \cdot$ P. Massoletti ${ }^{4} \cdot$ D. Lazzerini ${ }^{4} \cdot$ M.L. Brandi $^{5}$
}

Received: 18 November 2020 / Accepted: 6 May 2021 / Published online: 19 May 2021

(C) The Author(s) 2021

\begin{abstract}
Summary X-linked hypophosphataemia (XLH) is a lifelong condition. Despite the mounting clinical evidence highlighting the long-term multi-organ sequelae of chronic phosphate wasting and consequent hypophosphatemia over the lifetime and the morbidities associated with adult age, XLH is still perceived as a paediatric disease.

Introduction Children who have XLH need to transition from paediatric to adult healthcare as young adults. While there is general agreement that all affected children should be treated (if the administration and tolerability of therapy can be adequately monitored), there is a lack of consensus regarding therapy in adults.

Methods To provide guidance in both diagnosis and treatment of adult XLH patients and promote better provision of care for this potentially underserved group of patients, we review the available clinical evidence and discuss the current challenges underlying the transition from childhood to adulthood care to develop appropriate management and follow-up patterns in adult XLH patients.

Results and Conclusions Such a multi-systemic lifelong disease would demand that the multidisciplinary approach, successfully experienced in children, could be transitioned to adulthood care with an integration of specialized sub-disciplines to efficiently control musculoskeletal symptoms while optimizing patients' QoL. Overall, it would be desirable that transition to adulthood care could be a responsibility shared by the paediatric and adult XLH teams. Pharmacological management should require an adequate balance between the benefits derived from the treatment itself with complicated and long-term monitoring and the potential risks, as they may differ across age strata.
\end{abstract}

Keywords X-linked hypophosphatemia $\cdot$ Phosphate $\cdot$ Transition to adulthood $\cdot$ Clinical targets

Prof. Maria Luisa Bianchi passed away prior manuscript submission.

S. Giannini

sandro.giannini@unipd.it

1 Department of Medicine, Clinica Medica 1, University of Padova, Padova, Italy

2 Experimental Laboratory for Children's Bone Metabolism Research, Bone Metabolism Unit, Istituto Auxologico Italiano IRCCS, Milano, Italy

3 Department of Clinical Medicine and Surgery, Federico II University, Napoli, Italy

4 Medical Affairs, Kyowa Kyrin, Basiglio, (MI), Italy

5 Metabolic Bone Diseases Unit, Department of Surgery and Translational Medicine, University of Firenze, Firenze, Italy

\section{Introduction}

Phosphorus, a major component of all tissues, either in the form of inorganic or organic phosphate, is essential for many functions within the body, including energy storage and metabolism, cell signalling, enzyme activation, and nucleotide synthesis [1-3]. Therefore, the maintenance of extracellular and intracellular phosphate levels within a narrow range is both critical for the whole-body well-being and of crucial biological importance for bone health by virtue of its role in mature chondrocytes apoptosis in the growth plate [4]. Along with calcium, inorganic (i) phosphate (Pi) is the main constituent of hydroxyapatite, the bone mineral that strengthens the mechanical resistance of the organic matrix. Of note, in human adult body, about $90 \%$ of total phosphorus is stored in the bone as hydroxyapatite (calcium-phosphate) crystals whose structure is indicative of trabecular bone quality $[5,6]$. Regulation of phosphate balance relies on a fine-tuning 
cross-talk between the intestine and kidney $\mathrm{Na} / \mathrm{Pi}$ cotransporters, the intertwined hormonal pathways involving bone-derived fibroblast growth factor 23 (FGF23) and parathyroid hormone (PTH) and the endocrine negative feedback loops stemming from vitamin $\mathrm{D}$ and its active form, the $1,25(\mathrm{OH})_{2} \mathrm{D}$ [7-9]. Over the last decades, identification and molecular characterization of genetic defects leading to a number of rare inherited or acquired phosphate homeostasis disorders improved tremendously our understanding of bone health and paved the way for the development of therapeutic strategies restoring adequate phosphate levels [7-11].

Among the most common disturbances of phosphate homeostasis, X-linked hypophosphatemia (XLH) is the prototypic heritable renal phosphate wasting disorder, characterized by skeletal abnormalities of variable severity, growth retardation, rickets, and/or osteomalacia, bone pain, enthesopathy, osteoarthritis, spontaneous dental abscesses, hearing difficulties, and muscular dysfunction [12-15]. The variety of symptoms in XLH patients demands a lifetime multidisciplinary approach to patient care with the final aim of optimizing quality of life (QoL) and lifting the burden of such a debilitating life-long disease [16]. However, after the years of burdensome therapy during childhood to ensure adequate bone matrix mineralization, legs straightening, and growth and to prevent bowing, young adult patients with XLH often stop treatment and miss to be regularly monitored for the prevention and limitation of bone pain and fragility fractures, joint stiffness, and periodontal inflammation. Lessened parental surveillance, poor taste and gastrointestinal side effects of phosphate preparations, lack of convincing evidence of replacement therapy with active vitamin $\mathrm{D}$ and phosphate benefits in asymptomatic individuals, as well as some physician' advice of not continuing conventional treatment into adulthood may all have contributed to the low commitment to therapy commonly observed in young adults, especially if not managed by specialized centres [17]. Interestingly, while there is general agreement that all affected children should be treated (if the administration and tolerability of therapy can be adequately monitored), there is a lack of consensus regarding therapy in adults. Thus, in presence of uncertainty regarding indications and duration of the treatment in adults, the rationale for therapy is directed toward symptomatic osteomalacia and prevention or correction of fractures and stress fractures while leaving unresolved the issue whether therapy affects the other major adult sequelae of XLH, particularly enthesopathy and dental disease $[17,18]$.

Overall, there is an unrecognized burden of XLH in the adult population with patients being misdiagnosed during childhood or even remaining undiagnosed for a long time despite the occurrence of unresolved symptoms of their ongoing condition. In addition, owing to the rarity of XLH and the medical focus on the disease in children, it can therefore be difficult for adults to find clinicians who are familiar with
XLH and have previously treated someone with the disease as healthcare professionals outside of specialized centres receive little training on XLH.

To provide guidance in both diagnosis and treatment of adult XLH patients and promote better provision of care for this potentially underserved group of patients, we review the available clinical evidence and discuss the current challenges underlying the transition from childhood to adulthood care to develop appropriate management and follow-up patterns in adult XLH patients.

\section{Selection of evidence}

Papers for consideration for the present review were retrieved by a PubMed search, using different combinations of keywords (e.g. XLH AND adult AND hypophosphatemia), without limitations in terms of publication date and language. Papers were selected for inclusion according to their relevance for the topic, as judged by the authors.

\section{Epidemiology and burden disease of adult XLH}

XLH is caused by loss-of-function of PHEX (phosphateregulating gene with homology to endopeptidases on the $\mathrm{X}$ chromosome) and represents the most common cause of inherited phosphate wasting, with an incidence of 3.9 per 100,000 live births and a prevalence ranging from 1.7 per 100,000 children to 4.8 per 100,000 persons (children and adults) [19-22]. Overall, earlier studies on XLH prevalence, mostly using a mixture of hospital surveys and registry data, provided conflicting prevalence rates, due in part to differences in criteria for case identification and validation [19-21]. Furthermore, lack of accurate data for adults is compounded by the lack of any standard management for adults with XLH in terms of monitoring laboratory values, skeletal status, and other characteristics as well as frequent management in the primary care setting and even by a potential submerged prevalence in adult population indicating that the disease may also be underdiagnosed. A recent populationbased cohort study using a large primary care database in the UK estimated adult XLH prevalence being 15.7 per million and revealed a reduced survival among XLH cases relative to controls (with average age at death being approximately 8 years younger in cases relative to controls; 64 vs. 72 years) [23]. Nevertheless, prevalence and prognosis of adult XLH patients remain poorly described at both national and global level, and further studies are awaited to better characterize their phenotype and improve their care.

The burden of illness is substantial across the lifespan with an evolution of XLH burden from being mostly treatmentrelated in childhood to multi-factorial in adolescents with a 
rise in psychological burden, and higher impact on need for surgical and orthodontic interventions and lower physical function scores in adulthood compared to the general population $[24,25]$. The recent Burden of Disease Survey Study has expanded our understanding of XLH from both the paediatric and adult patient perspective and revealed that nearly all adults experienced bone or joint pain, joint stiffness, restricted range of motion, frequent dental abscesses, as well as substantial complications stemming from unresolved childhood symptoms and emerging as the disease progresses [26]. Of note, a significant morbidity is associated with the condition as patients get older, particularly with osteoarthritis, enthesopathy, or insufficiency fractures. As nearly half of adults undergo some form of corrective surgery of skeletal abnormalities originated during skeletal growth and face up to frequent orthopaedic and orthodontic surgeries and specialist visits, the burden posed by XLH from a socio-economic perspective is expected to be substantial although available data are scant. Thus, quantification of the economic burden of XLH is warranted in order to further highlight the unmet needs in XLH.

\section{Adult XLH profile: clinical presentation and diagnosis}

Owing to the rarity of XLH and the diversity of clinical manifestations, diagnosis is often delayed thus posing patients at higher risk of long-term complications and inadequate treatment. The clinical features, biochemical alterations, and radiographic manifestations that should prompt a diagnostic workup in adult patients encompass short stature, osteomalacia, bone pain, osteoarthritis, hypophosphatemia, elevated alkaline phosphatase (ALP), pseudo-fractures, stiffness, and enthesopathies [27-31]. Of note, in presence of a negative family history, documented in up to one-third of reported patients, a mutational analysis of the PHEX gene can provide diagnosis confirmation in $\sim 70-90 \%$ of cases $[32,33]$.

Adults with XLH have a significantly reduced final height compared to reference standards and appear disproportionate, with leg length scores being significantly lower than those for sitting height and relatively short arms compared to height [28]. Lower-extremity bowing is also documented with genu varum (outward bowing of the lower leg) or genu valgus (inward bowing) frequently occurring [34]. Adult patients report joint pain (more frequent in knee and followed by painful ankles and hip pain) and arthritis, mostly at age older than 40 years, and experience more frequently spinal stenosis requiring decompression or joint disease requiring knee or hip replacement $[28,30]$. Symptoms such as pain and fatigue can be quite common with 90.4 and $86.0 \%$ of patients reporting them at the time of evaluation [29].

Bone remodelling continues throughout life, and in patients with XLH, this means that new bone being laid down may not be fully mineralized, leading to a reduction in bone quality whose clinical effects in adult life will not become apparent until it reaches a clinically meaningful threshold and may contribute to the increased risk in pseudo-fractures, fractures and impaired fracture healing observed in adult patients. A recent survey suggested age-specific differences in bone fractures incidence with femoral fractures first occurring at 29.5 years of age, tibia/fibula and hand/wrist first presenting at 21.5 and 22.4 years of age, respectively, feet fractures at 37.5 years of age, and hip mostly presenting at 40 years of age [35]. Enthesopathy and osteoarthritis of the spine and joints are among the most significant complications and complaints of adult patients affected by XLH and are common radiographic features [27]. Short case series and case reports have also provided evidence of a wide range of spinal manifestations in adult XLH patients including ossification of the paraspinal ligaments, thickening of the laminae, calcification of the discs, and facet joint hypertrophy. Of note, spinal canals of XLH patients were found to be narrower than those of normal individuals, thus contributing to a propensity to a spinal stenosis $[30,36]$. Furthermore, a radiologic survey of 38 untreated adults revealed flaring of the iliac wings, trapezoidal distal femoral condyles, shortening of the talar neck, and flattening of the talar dome [37]. Finally, orthopantograms frequently show root dysplasia and enlarged pulp chambers [38].

The biochemical hallmarks of XLH are hypophosphatemia due to renal phosphate wasting and increased alkaline phosphatase [ALP in children, bone alkaline phosphatase (BAP) in adults] levels [17, 22, 27]. Inappropriately high FGF23 levels might further contribute to diagnostics but are costly and often not readily available; thus, in resource limited settings, clinicians may refer to a biochemical diagnostic work-up as previously described [27], while assessing intact FGF23 to confirm clinical diagnosis if genetic analysis is not available.

Adult XLH patients may also present with extra-skeletal manifestations including dental complications (e.g. periodontitis, tooth decay and dental abscesses), nephrocalcinosis, hyperparathyroidism requiring parathyroidectomy (when not efficiently managed with cinacalcet [39-45]), cardiovascular abnormalities (i.e. early-onset hypertension, left ventricular hypertrophy [46, 47]), and hearing loss [48]. Of note, hearing issues are more frequently seen in adults than children (48$82 \%$ vs. $9 \%$ ) with sensorineural hearing loss, often asymmetric, being most prominent, although a mixed picture, thought to be caused by endolymphatic hydrops, can also be seen [30]. Importantly, patients with XLH may frequently require hearing aids and are advised to avoid noise exposure or administration of ototoxic drugs. In contrast to systemic effects, dental symptoms may not be FGF23-driven but directly caused by local effects of extracellular matrix components due to impaired PHEX function [49]. Nephrocalcinosis has not been reported in untreated XLH patients and is widely considered to be a result of conventional therapy associated with active 
vitamin D dosage [13]. Of note, reports of cardiovascular abnormalities and hypertension in patients with XLH are rare, inconsistent, and considered to be side effects of conventional therapy and/or FGF23-driven increased renal sodium reabsorption $[13,47,50]$. It has been hypothesized that extraskeletal complications may occur more frequently in presence of extremely high FGF23 levels as documented in chronic kidney disease (CKD) and less when FGF23 elevation is not marked as often observed in XLH patients [51].

It has been reported that some adult patients may not have clinical manifestations at all and consequently considered not candidate to conventional therapy $[16,27]$. However, the underlying chronic hypophosphatemia still drives disease progression, although at slower rate, and requires a careful monitoring of the long-lasting sequelae of prolonged hypophosphatemia that poses a significant burden on XLH patients' QoL [29]. Finally, it should be acknowledged that hypophosphatemia can be an incidental finding as it may be commonly missed due to nonspecific signs and symptoms. For example, children and adult patients might present with spontaneous endodontic infections on apparently intact teeth that might be asymptomatic for months or years [1, 27, 52].

As recently suggested in evidence-based recommendations, the diagnosis of XLH in adults should be suspected in case of presence or history of lower limb deformities and/or typical clinical or radiological signs of osteomalacia (including pseudofractures, early osteoarthritis, and enthesopathy) in the context of low levels of serum phosphate associated with renal phosphate wasting. Figure 1 illustrates our proposed diagnostic algorithm to identify adult XLH patients. Among the biochemical abnormalities that may drive the suspicion of a potential hypophosphatemic disease, we include first of all low serum phosphate levels, elevated BALP, unmodified PTH levels (in absence of a deranged PTH secretion, that can be present, as previously mentioned, in some patients) and normal or slightly lower levels of serum calcium; all parameters assessed upon fasting. If BALP measurement is not feasible, ALP, $\gamma$-glutamyl transferase $(\gamma$-GT), aspartate aminotransferase (AST/GOT), and alanine aminotransferase (ALT/GPT) dosing may be considered an option. In case of suspicion of renal phosphate wasting diseases, the first step must be to determine whether urinary phosphate excretion is appropriate, followed by careful medical history and objective examination to help distinguish the various forms. Indices of phosphate excretion, such as tubular maximal reabsorption of phosphate adjusted for glomerular filtration rate (TmPi/GFR) as well as measurement of fractional phosphate excretion (FEPi), allow to determine if renal loss is or is not a factor in causing hypophosphatemic state. The resulting values may inform clinicians about the presence of either renal phosphate wasting or reduced intestinal phosphate intake and/or absorption. Renal phosphate wasting might be the result of either primary tubular defects or high levels of circulating FGF23 in both cases inherited or acquired (Fig. 1).

To date, here we focus on the long-lasting and chronic, sequelae of prolonged hypophosphatemia as seen in XLH patients whose hypophosphatemia is due to increased renal phosphate wasting with a resulting increased alkaline phosphatase [ALP in children, bone alkaline phosphatase (BAP) in adults] levels. A comprehensive review of the multiple mechanisms of hypophosphatemia is beyond the scope of our work, and to this end, we refer the interested reader to previous publications $[1,4,10]$.

\section{Causes of chronic hypophosphatemia}

The causes of chronic hypophosphatemia encompass many conditions, namely, a reduced phosphate absorption (due to, e.g. a vitamin D deficiency or Vitamin D resistance), an increased urinary excretion of phosphate PTH-dependent (either stemming from primary or secondary hyperparathyroidism), FGF23-dependent hypophosphatemia either acquired [such as tumour-induced osteomalacia (TIO) or iron-induced hypophosphatemia following intravenous administration of ferric carboxymaltose] [53, 54], or genetic and tubulopathies [including hereditary hypophosphatemic rickets with hypercalciuria (HHRH), hypophosphatemia and nephrocalcinosis (NPHLOP1), generalized proximal tubulopathies (i.e. acquired and genetic Fanconi syndrome)]. Vitamin D deficiency and hyperparathyroidism (primary, secondary, and post-renal transplant hyperparathyroidism) stand as the most frequent causes of hypophosphatemia that should be searched for and adequately treated before undertaking extensive investigations. All FGF23-dependent causes display the same biochemical features and are biochemically indistinguishable; thus, careful evaluation of family and personal history, childhood onset of symptoms, associated clinical and radiological features, may help to distinguish between acquired and genetic causes [54].

Genetic forms FGF23-dependent include XLH, autosomal dominant or recessive hypophosphatemic rickets (ADHR and ARHR), and polyostotic fibrous dysplasia, hypophosphatemic rickets with hyperparathyroidism and non-lethal Raine syndrome [16, 53, 54]. Patients with ADHR mutations may exhibit either early or delayed onset of disease presentation making the differential diagnosis difficult with acquired FGF23-dependent hypophosphatemic rickets such as TIO. Of note, while current evidence suggests that ADHR patients may not display enthesopathy as seen in XLH [54], two reports of families with ARHR1 and ARHR2 displaying severe mineralizing enthesopathy and calcifications of the spinal ligaments suggest that the enthesopathy may be not unique to XLH [55]. Fibrous dysplasia patients sometimes suffer from concomitant hypophosphatemic rickets/osteomalacia, resulting from phosphate wasting and the 


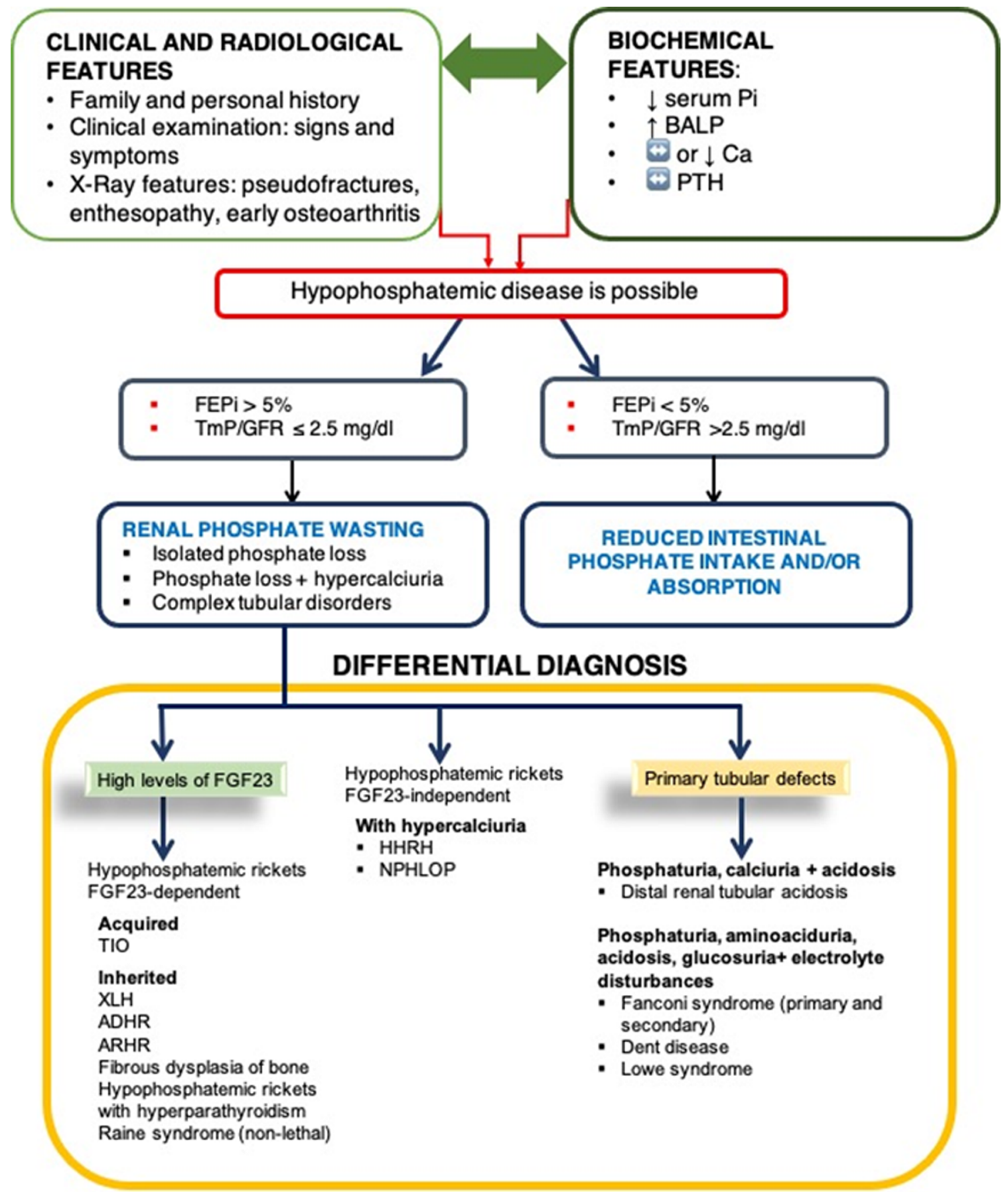

Fig. 1 Algorithm for the diagnosis of an adult with radiological and clinical features suggestive of XLH and presenting with hypophosphatemia. Biochemical work-up should be carried out upon fasting. This algorithm requires prior exclusion of co-morbidities causing hypophosphatemia including sepsis, heart stroke, hepatic coma, myeloma, diabetic ketoacidosis, alcohol abuse, refeeding after prolonged malnutrition, severe respiratory alkalosis, and hyperparathyroidism. Increased renal phosphate secretion may be secondary to PTH-mediated mechanism and in presence of elevated PTH levels, abnormalities in vitamin D metabolism, calcium deficiency, and primary hyperparathyroidism should be excluded. Furthermore, this algorithm requires prior exclusion of co-administration of drugs causing hypophosphatemia including diuretics and antibiotics (that are found to induce hypophosphatemia through impairment of renal phosphate transport), antacids (by reducing intestinal absorption), bisphosphonates (trough secondary hyperparathyroidism), insulin (via shifts of extracellular phosphate into cells), ferric carboxymaltose (by transiently rising the levels of intact FGF23), and canagliflozin (that may induce hypophosphatemia as a result of euglycemic diabetic ketoacidosis). Abbreviations: ADHR, autosomal dominant hypophosphatemic rickets; BALP, bone alkaline phosphatase; ARHR, autosomal recessive hypophosphatemic rickets; $\mathrm{Ca}$, calcium; FEPi, fractional phosphate excretion; GFR, glomerular filtration rate; HHRH, hereditary hypophosphatemic rickets with hypercalciuria; NPHLOP, nephrolithiasis; PTH, parathyroid hormone; TIO, tumour-induced osteomalacia; TmPi, tubular maximal reabsorption of phosphate; XLH, X-linked hypophosphatemia. ARHR encompasses ARHR type 1, type 2, and type 3 (also known as Raine syndrome) 
FGF23 originating from dysplasic tissue might cause hypophosphatemia in these patients who, similarly to XLH patients, can display normal levels of $1,25(\mathrm{OH}) 2 \mathrm{D}$ [31]. Nevertheless, one should also take into consideration that XLH patients may display both normal and inappropriately low $1,25(\mathrm{OH}) 2 \mathrm{D}$ serum concentrations. Clinical and biochemical features of ADHR and ARHR are similar to XLH, with the exception of a relatively higher bone mineral density (BMD) in ADHR [56]. FGF23-related hypophosphatemia can also be observed in patients with non-lethal Raine syndrome who exhibit a generalized osteosclerosis with periosteal bone formation $[27,54,57]$. Of note, Raine syndrome differs from $\mathrm{XLH}, \mathrm{ADHR}$, and ARHR types 1 and 2 in that it is associated with intracerebral calcifications and a higher incidence of dental abnormalities [54]. Overall, a mutational analysis of the PHEX gene may ultimately confirm XLH diagnosis in the aforementioned cases. Of note, beyond the aforementioned conditions, there are also other FGF23-related hypophosphatemic diseases such as osteoglophonic dysplasia, Jansen-type metaphyseal chondrodysplasia, and linear nevus sebaceous syndrome [58].

Hypophosphatemia due to renal wasting can be associated with renal tubular disorders that differ from other hypophosphatemic rickets in that $1,25(\mathrm{OH}) 2 \mathrm{D}$ serum concentrations are elevated thus resulting in an increased intestinal calcium absorption and subsequently hypercalciuria. In the context of tubulopathies, it is important to consider HHRH (characterized by reduced renal phosphate reabsorption, hypophosphatemia, and rickets, with high $1,25(\mathrm{OH}) 2 \mathrm{D}$ levels resulting in hypercalciuria), NPHLOP, and NHERF1 mutations or to exclude the presence of Fanconi syndrome (either acquired or genetic). Defective handling of phosphate, glucose, and amino acids is the cardinal feature of the Fanconi; thus, common laboratory findings include glucosuria with a normal serum glucose, hypokalaemia, hypophosphatemia, hypouricemia, progressive renal insufficiency, hyperaminoaciduria, renal sodium and potassium wasting, acidosis, uricosuria, and low molecular weight proteinuria. The leading genetic cause of Fanconi syndrome is cystinosis followed by the Dent disease types 1 and 2 with the latter being caused by mutations in the same gene that causes Lowe syndrome, a rare multisystem disorder. While the Fanconi syndrome should be excluded by looking for phosphaturia and calciuria plus acidosis, and Dent and Lowe diseases can be excluded by looking for phosphaturia, aminoaciduria, acidosis, glucosuria, and electrolyte disturbances [53, 54].

To date, drug-induced hypophosphatemia (i.e., diuretics, bisphosphonates, antibiotics, insulin, canagliflozin, ferric carboxymaltose, antacids) must also be considered in adult patients with low phosphate levels $[31,54,59]$. Of note, diuretics and antibiotics are found to induce hypophosphatemia through impairment of renal phosphate transport, antacids by reducing intestinal absorption, bisphosphonates trough secondary hyperparathyroidism, insulin via shifts of extracellular phosphate into cells, and ferric carboxymaltose by transiently rising the levels of intact FGF23; finally, canagliflozin may induce hypophosphatemia as a result of euglycemic diabetic ketoacidosis [54, 59].

Finally, XLH symptoms may lead to misdiagnosis, especially in not specialized centres. For example, adults may be mistakenly diagnosed with disorders responsible for early severe stiffness and/or with enthesopathies, such as ankylosing spondylitis and diffuse idiopathic skeletal hyperostosis [60]. Of note, patients suffering from axial spondyloarthritis or aged patients with diffuse idiopathic skeletal hyperostosis display radiological phenotype of enthesopathies and paravertebral ossification similar to XLH [29].

\section{XLH burden on quality of life: implications in adult daily life}

XLH adults report a lower QoL than the general population, and their QoL is similar to that of patients with chronic diseases such as rheumatoid arthritis [29]. Of note, QoL of adult $\mathrm{XLH}$ patients is more severely impaired than that of young adults affected with axial spondyloarthritis [29]. Age, female gender, presence of structural lesion, fatigue, and enthesopathies appear to be significantly associated with a worse QoL [29, 52]. Furthermore, secondary complications including early development of osteoarthritis substantially impair QoL and cause significant clinical burden [16]. When children transition to being adolescents, the burden of XLH becomes increasingly multifactorial and a noticeable presence of psychological burden has been documented with more frequently observed anxiety, distress, and low self-esteem. Compared to children, adolescents and young adult patients suffer more frequently from being fully reliant on others for their daily living or caregiving duties and from being often overwhelmed by the disease-related healthcare utilization and costs (countless surgeries, dental issues, specialist visits, weekly use of analgesics, etc.) $[25,26]$. A large proportion of patients quit their jobs or even retire earlier as they no longer cope with the pain of XLH as well as many patients require more frequently sick leave due to fatigue [24]. In line with earlier observations [26, 29, 61], a recent European multi-country qualitative study suggested that pain was the most relevant symptom of adult XLH, leading to difficulties walking/running, standing, sitting for long, or specific types of movements. A variable degree of disability ranging from minimal to being confined to a wheelchair has been reported with mobility problems heavily affecting daily activities, such as work, housework, and shopping [17, 22, 30]. Furthermore, 
pain had a considerable psychological impact, with people commonly describing it as affecting their mood/mental health (e.g. feeling down or depressed). Pain also impacted relationships, leisure activities and social life, as it often affected motivation to go out or led to plans being cancelled [24]. XLH patients may experience an additional psychological burden due to their physical appearance and the hereditary and progressive nature of their condition with the majority being concerned about their future. The most common concerns included disease progression and subsequent inability to work as well as symptom deterioration over time with consequent worse physical functioning and further need of undergoing surgical procedures. Nevertheless, the commonly reported mental resilience resulting from XLH might reduce the perceived impact of their condition $[24,25]$.

\section{Transition to adulthood and adult healthcare for patients with XLH: current practice and challenges}

XLH is a life-long debilitating disease with adults continuing to experience the effects of unresolved childhood symptoms and the metabolic and endocrine consequences of a chronic phosphate wasting and hypophosphataemia. To date, adult symptoms may result from persistent unresolved complications from childhood (lower extremity deformities, short stature) or may appear during adulthood such as enthesopathy, early onset osteoarthritis, hearing loss, and pseudo-fractures $[17,26]$. Despite this mounting evidence, XLH is currently regarded as a paediatric disease and more frequently managed by paediatricians even in adult age. As suggested by a recent survey carried out in Italy, half of patients reaching adult age continue to be followed by paediatric centres with the other half being referred to adult endocrinologists, nephrologists, and rheumatologists [62]. While multiple reports endorse the standard that paediatric providers should prepare their patients for transfer to adult care, there are no generally accepted guidelines to assure smooth transfer from paediatric to adult centre. As documented for other rare diseases, without adequate support, adolescents transitioning from the paediatric to the adult health care system are at increased risk of poor health outcomes [63] thus emphasizing the need of an effective, multidisciplinary, and well-supported transition.

While the attention on issues surrounding the care transition in rare disease patients is growing, we face a general lack of epidemiological data quantifying the impact of this phenomenon and a frequent underestimation of adult patients due to under-ascertainment of cases or loss to follow-up. With regard to XLH, a smooth transition to adulthood care can be hampered by the limited evidence of beneficial effects of phosphate and vitamin D-based conventional oral treatment in asymptomatic adult patients that may have driven therapy discontinuation, either spontaneously or following doctor's advice [27]. Comorbidities that can make transition to independence more difficult, notably mental and behavioural health and cognitive and educational challenges, poor understanding of current care and its benefits, as well as the attachment to a long-term paediatric care team may all contribute as further barriers to a smooth transition $[64,65]$. The available medical treatment in adults is quite similar to that in children, including phosphate and active vitamin D substitution that, despite their wide use, displays several limitations included limited compliance and adverse effects such as secondary hyperparathyroidism, nephrocalcinosis, nephrolithiasis, hypercalcemia, and gastrointestinal symptoms (bloating, nausea and diarrhoea) [43]. Although continuing conventional therapy during adulthood has proved to be beneficial for oral health with patients treated their entire adult life having lower odd ratios for having more than five dental abscesses compared to those receiving treatment for shorter period [18], little evidence suggests that starting or continuing conventional therapy improves outcomes. To date, there is controversy regarding continuing conventional treatment into adulthood due to both its poorly documented efficacy and the related unfavourable effects that may limit patients' compliance [18].

Furthermore, prospective studies reported that continuing conventional medical therapy in adulthood, although associated with increased bone resorption, did not promote or prevent loss of bone mass as evidenced from the stable areal bone mineral density of the hip and spine in XLH patients [66] and can improve, but not completely reverse, histomorphometric parameters of osteomalacia [67]. To date, in the largest survey of patients with XLH, only half of adult patients were receiving oral phosphate [26]. Conventional treatment with daily active vitamin D and twice-daily oral phosphate supplements appears burdensome for many adult patients, due to both inconvenient dosing regimens and unpleasant taste. Although less frequent dosing might improve adherence in adolescents [27] and more palatable effervescent oral formulations of phosphate have been made available to patients, adult patient adherence to conventional therapy appears low thus leading to a very limited engagement to the currently available medications and subsequently treatment discontinuation. Therefore, ensuring that as many patients as possible can benefit from continuing therapy across adolescence and during adulthood is an unmet need for the XLH field thus demanding new therapeutic approaches to be investigated. In this context, new evidence is emerging on the therapeutic potential of interventions targeting FGF23 in adults to manage hypophosphatemia by improving fracture healing and key histomorphometric parameters (osteoid volume/bone volume, osteoid surface/bone surface, and osteoid thickness) while reducing patient-reported stiffness compared to placebo [68, 69]. 
Overall, a smooth transition to adulthood care requires efforts from both patient and paediatric care provider with the latter facilitating referral to adult specialized teams, demands increased awareness of the lifelong sequelae of a chronic phosphate wasting and its multi-organ complications that need to be timely addressed in adults as well as optimization of the available treatment options to promote patient engagement to a lifelong management.

\section{Clinical therapeutic targets in adult patients with X-linked hypophosphatemia}

Evidence in recent years points to phosphate as the main culprit in XLH-associated skeletal and metabolic abnormalities thus supporting the rationale of targeting serum phosphate level to monitor treatment efficacy over time and across age strata. Phosphate level normalization and the subsequent phosphate homeostasis restoration are relevant both in the context of bone health and for protecting systemic wellbeing given the detrimental consequences of severe hypophosphatemia including haemolysis, rhabdomyolysis, respiratory failure, and left ventricular dysfunction $[3,10$, 31]. However, the current goal of treatment of XLH in adult patients is not only to normalize the serum phosphate levels (by means of $\mathrm{TmPi} / \mathrm{GFR}$ monitoring and subsequent restoration of renal Pi reabsorption) but even to reduce pain and osteomalacia extent, to improve the symptoms, to accelerate fracture healing, and facilitate surgical recovery. Each of the aforementioned treatment goals rely on different monitoring approaches that are endowed of distinct reliability and sensitivity.

A long-standing controversy exists regarding pain assessment and, as pain perception is highly individual and subjective in nature, it may be adequately and reliably assessed only through patient report. This is particularly relevant in a condition like XLH that is characterized by internal experiences of the patient such as pain and stiffness and where patientreported data form an important part of treatment efficacy evaluation [60]. In this context, the short-form of the Brief Pain Inventory (BPI) questionnaire and the full Western Ontario and McMaster Universities Osteoarthritis Index (WOMAC) questionnaire, designed for the assessment of lower-extremity pain and function in osteoarthritis of the knee or hip and extensively employed in low back pain, rheumatoid arthritis, fibromyalgia, and lupus erythematosus, was found to be suitable endpoints in XLH clinical trials in adults [68, 69]. Such tools proved to be able to capture the plethora of physical functioning limitations observed in XLH, particularly in terms of difficulty in walking, standing, and sitting and a reduced range of motion that present as direct result of the pain and stiffness experienced by the patients in relation to their XLH.
Osteomalacia is the hallmark of XLH in adult patients and is characterized by mineralization defects that impair both bone quality and remodelling; thus, restoring phosphate homeostasis may reduce osteomalacia extent and its contribution, along with skeletal deformities, to the higher risk of pseudofractures. Bone biopsies are regarded as too invasive as routine tools to measure such therapeutic response, and there are no currently good non-invasive markers able to quantify the extent of the osteomalacia before and after treatment [22]. However, as documented following 48 weeks of treatment with burosumab, a fully human monoclonal antibody against FGF23, adult XLH patient did experience an improvement in osteomalacia and an increase of biochemical markers of bone remodelling including BALP, procollagen type $1 \mathrm{~N}$-propeptide (P1NP, marker of bone formation), and carboxyterminal cross-linked telopeptide of type I collagen (CTx, marker of bone resorption) that were found useful in providing an early assessment of treatment efficacy [69].

In agreement with previous reports in paediatric patients, treatment with burosumab rapidly and significantly increased $\mathrm{TmPi} / \mathrm{GFR}$ with raised levels of serum phosphate in the lower part of normal range and increased $1,25(\mathrm{OH})_{2} \mathrm{D}$ levels in adulthood along with a sustained improvement in key musculoskeletal impairments upon both 24 and 48 weeks of treatment $[68,69]$. Adults with XLH, taking oral phosphate and active vitamin D metabolites or analogs prior study enrolment, when randomized to receive burosumab for 6 months, experienced a rapid normalization of serum phosphate concentration, 17-fold greater likelihood of fracture healing and marked improvement in functional exercise capacity compared to those receiving placebo. When the treatment with burosumab was extended, in an open-label fashion, to additional 6 months, serum phosphate levels remained normal in $83.8 \%$ of patients along with a proportion of fully healed fractures and pseudofractures progressively increased, with $80 \%$ achieving partial or complete healing by week 48 , thus supporting sustained efficacy of burosumab in normalizing phosphorus homeostasis and positively impacting hypophosphataemia clinical consequences [70].

Secondary hyperparathyroidism with disrupted physiological regulation of parathyroid function is frequent in adult XLH patients. Young-onset hypercalcemic hyperparathyroidism, resulting from parathyroid adenomas or hyperplasia, worsens hypophosphatemia and leads to renal and skeletal complications. Thus, prevention of secondary hyperparathyroidism is an important goal in management of both paediatric and adult XLH patients $[52,71,72]$. To date, no evidence of tertiary hyperparathyroidism was reported in adult patients (who had serum calcium $\geq 10.8 \mathrm{mg} / \mathrm{dL}$, serum intact $\mathrm{PTH} \geq 2.5$ fold the upper limit of normal and reported use of PTHsuppressing medication or positive history for fracture) following 48 weeks of treatment with burosumab [70]. 
Management of hyperparathyroidism is relevant if one considers the extremely low levels of $1,25(\mathrm{OH})_{2} \mathrm{D}$ that impact on bone health and PTH levels by FGF23dependent and independent mechanisms. To this end, it would be advisable to monitor serum levels of $25(\mathrm{OH})$ vitamin $\mathrm{D}$ [73] both in patients receiving human monoclonal antibody against FGF23 and in those treated with conventional therapy. In the former, a reduction in serum $25(\mathrm{OH})$ vitamin $\mathrm{D}$ was reported over 24 weeks of treatment in $8 \%$ of patients probably as result of increased conversion in $1,25(\mathrm{OH})_{2} \mathrm{D}$; of note, native vitamin $\mathrm{D}$ supplement was able to restore $25(\mathrm{OH})$ vitamin D levels $[68,74]$. Finally, in those treated with conventional therapy, particularly in those for whom a long-term immobilization is anticipated, a reduction of the doses of active vitamin D can be desirable to prevent hypercalcemia and hypercalciuria [27].

With regard to dosing regimens, as recalled in [27], as of today no consensus has been reached on the optimal dose of oral phosphate. Furthermore, a high variability in doses and treatment regimens exists with conventional therapy based on patients' age, phenotype severity, potential unfavourable effects, or even medical expertise. Overall, the treatment scenario in the adult population comprises a plethora of settings including untreated patients, those on therapy since childhood, those receiving vit $\mathrm{D}$ only or vit $\mathrm{D}$ combined with oral phosphate.

\section{Follow-up in adult patients with XLH}

Disease progression, occurrence of complications related to unresolved childhood symptoms, and potential adverse effects arising from conventional therapy administration demand frequent follow-up and monitoring of adult patients. Follow-up in adults includes clinical, biochemical, and radiologic assessments to adjust treatment or dosing regimen, to treat the adultonset symptoms in a timely manner and balance skeletal healing with complications such as hyperparathyroidism and nephrocalcinosis. In this context, imaging and instrumental assessment such as brain MRI, cardiac and renal ultrasound, dental orthopantomogram may be of help while dual energy$\mathrm{X}$-ray absorptiometry (DXA) scan seems not playing any role in follow-up when used to measure bone mineral density [16, 27]. Of note, brain MRI can be performed when recurrent headaches, declining school/cognitive performances, or neurological symptoms are reported or in case of craniosynostosis or clinical signs of intracranial hypertension. A recommended follow-up plan for adult patients with XLH is presented in Table 1. Currently, there is no specific recommended

Table 1 Follow-up plan for adult patients with XLH. Elaborated from data in [27, 52]

\begin{tabular}{|c|c|}
\hline Monitoring & Interval \\
\hline \multicolumn{2}{|l|}{ Clinical examination } \\
\hline Record of history of headache, oral manifestations, fatigue, pseudo-fractures, musculoskeletal pain & 6-monthly if treated, 12-monthly if untreated \\
\hline Search for evidence of hearing loss & 6-12 monthly \\
\hline Neurological evaluation & 12-monthly \\
\hline \multicolumn{2}{|l|}{ Lab monitoring } \\
\hline $\begin{array}{l}\text { Serum calcium, phosphate, } \mathrm{PTH} \text {, creatinine, } 25(\mathrm{OH})_{2} \text { vitamin } \mathrm{D}, \mathrm{ALP}, 1,25(\mathrm{OH})_{2} \mathrm{D}, 24 \mathrm{~h} \text {-urine } \\
\text { calcium and phosphate, TmPi/GFR }\end{array}$ & 6-12 monthly \\
\hline \multicolumn{2}{|l|}{ Special monitoring } \\
\hline BALP, P1NP, CTx & 6-monthly/once-year \\
\hline \multicolumn{2}{|l|}{ Functional monitoring } \\
\hline 6-min walk test & 12-monthly \\
\hline \multicolumn{2}{|l|}{ Quality of life monitoring } \\
\hline BPI and WOMAC & 12-monthly \\
\hline \multicolumn{2}{|l|}{ Dental monitoring } \\
\hline Periodontal risk assessment and supra-gingival and subgingival debridement & 6-monthly \\
\hline Dental orthopantomogram & 6-monthly \\
\hline \multicolumn{2}{|l|}{ Imaging } \\
\hline $\begin{array}{l}\text { Cranial MRI if suggestive of craniosynostosis or when recurrent headaches, declining } \\
\text { school/cognitive performances or neurological symptoms are reported }\end{array}$ & 6-12-monthly \\
\hline Cardiac echocardiogram if hypertensive & 12-monthly \\
\hline Kidney ultrasound for nephrocalcinosis/hypercalciuria & 12-monthly \\
\hline
\end{tabular}

$A L P$, alkaline phosphate; $B A L P$, bone alkaline phosphate; $B P I$, brief pain inventory; $C T x$, carboxyterminal cross-linked telopeptide of type I collagen; $M R I$, magnetic resonance imaging; $P 1 N P$, procollagen type $1 \mathrm{~N}$-pro-peptide; $P T H$, parathyroid hormone; $T m P i / G F R$, tubular maximal reabsorption of phosphate/glomerular fraction rate; WOMAC, Western Ontario and McMaster Universities Osteoarthritis Index 
follow-up plan for patients treated with burosumab. Pending further clinical studies on long-term efficacy and safety with burosumab in adult XLH patients, we refer the interested reader to what is currently suggested by Haffner et al. [27]. Nevertheless, as suggested by Saraff et al. [75], the most useful biomarkers for treatment monitoring include serum ALP, TmPi/GFR, and knee and wrist X-rays. Overall, XLH management requires a fine-tuning balance between the benefits of treatment with complicated monitoring and potential undesirable effects including vitamin D deficiency and secondary or tertiary hyperparathyroidism.

\section{Conclusions and perspectives}

Despite the mounting clinical evidence highlighting the longterm multi-organ sequelae of chronic phosphate wasting and consequent hypophosphatemia over the lifetime and the morbidities associated with adult age, XLH is still perceived as a paediatric disease. Thus, XLH is frequently managed by paediatricians even in adult age with time-evolving consensusbased indications for its management and follow-up that are subjected to frequent revisions pending further clinical evidence as bases to update recommendations [27]. Such a multi-systemic lifelong disease would demand that the multidisciplinary approach, successfully experienced in children, could be transitioned to adulthood care with an integration of specialized sub-disciplines to efficiently control musculoskeletal symptoms while optimizing patients' QoL. By virtue of the clinical relevance of genetic testing in adults' diagnosis and subsequent management, inclusion of a geneticist in the multidisciplinary team is desirable to provide preconception counselling when needed. Overall, it would be desirable that transition to adulthood care could be a responsibility shared by the paediatric and adult XLH teams. Pharmacological management should require an adequate balance between the benefits derived from the treatment itself with complicated and long-term monitoring and the potential risks, as they may differ across age strata. However, the response to conventional treatment appears unsatisfactory in many patients with no substantial effect on the course of the most disabling feature of adult XLH, i.e. enthesopathy. Furthermore, given the variety of hypophosphatemia-derived complications [76] and their impact on natural history of the disease as well as the feedback loops affected by either phosphate or active vitamin D supplementation, several research priorities exist. Thus, we need to expand our knowledge on adult XLH phenotype and their responsiveness to therapy by developing a comprehensive registry in XLH as well as clinical and/or radiological scores to evaluate treatment efficacy and tolerability. Furthermore, studies directed toward adult morbidities in XLH patients would be important to confirm the effectiveness of continued treatment through adult years although they may require a lengthy follow-up period to capture sufficient data for these age-dependent outcomes to develop. Considerable hope is therefore being placed in the recent evidence supporting the use of monoclonal antibody against FGF23 in adults $[68,69]$ in view of further evidence from both longterm outcomes (up to 96 weeks) and real-life setting.

Acknowledgements Writing and editing assistance was provided by EDRA SpA, with the helpful support of Chiara Degirolamo, $\mathrm{PhD}$, and supported by Kyowa Kirin.

Author contribution All authors contributed equally to this review by writing the text and producing personal data and illustrations; S. Giannini coordinated the review.

Funding This work was supported by Kyowa Kirin Italia.

\section{Declarations}

Conflict of interest Sandro Giannini, Maria Luisa Bianchi, Domenico Rendina, Maria Luisa Brandi declare that they have no conflict of interest. Pierfrancesco Massoletti and Debora Lazzerini are employees of Kyowa Kirin Italia.

Open Access This article is licensed under a Creative Commons Attribution-NonCommercial 4.0 International License, which permits any non-commercial use, sharing, adaptation, distribution and reproduction in any medium or format, as long as you give appropriate credit to the original author(s) and the source, provide a link to the Creative Commons licence, and indicate if changes were made. The images or other third party material in this article are included in the article's Creative Commons licence, unless indicated otherwise in a credit line to the material. If material is not included in the article's Creative Commons licence and your intended use is not permitted by statutory regulation or exceeds the permitted use, you will need to obtain permission directly from the copyright holder. To view a copy of this licence, visit http:// creativecommons.org/licenses/by-nc/4.0/.

\section{References}

1. Imel EA, Econs MJ (2012) Approach to the hypophosphatemic patient. J Clin Endocrinol Metab 97:696-706. https://doi.org/10. 1210/jc.2011-1319

2. Penido MG, Alon US (2012) Phosphate homeostasis and its role in bone health. Pediatr Nephrol 27:2039-2048. https://doi.org/10. 1007/s00467-012-2175-z

3. Manghat P, Sodi R, Swaminathan E (2014) Phosphate homeostasis and disorders. Ann Clin Biochem 51:631-656. https://doi.org/10. $1177 / 0004563214521399$

4. Christov M, Juppner H (2018) Phosphate homeostasis disorders. Best Pract Res Clin Endocrinol Metab 32:685-706. https://doi. org/10.1016/j.beem.2018.06.004

5. Michigami T, Ozono K (2019) Roles of phosphate in skeleton. Front Endocrinol 10:180. https://doi.org/10.3389/fendo.2019. 00180

6. Rollo JMDA, Boffa RS, Cesar R, Schwab DC, Leivas TP (2015) Assessment of trabecular bones microarchitectures and crystal structure of hydroxyapatite in bone osteoporosis with application 
of the Rietveld method. Procedia Engineering 110:8-14. https://doi. org/10.1016/j.proeng.2015.07.003

7. Shimada T, Kakitani M, Yamamzaki Y, Hasegawa H, Takeuchi Y, Fujita $\mathrm{T}$ et al (2004) Targeted ablation of Fgf 23 demonstrates an essential physiological role of FGF23 in phosphate and vitamin D metabolism. J Clin Invest 113:561-568. https://doi.org/10.1172/ JCI19081

8. Lanske B, Razzaque MS (2014) Molecular interactions of FGF23 and PTH in phosphate regulation. Kidney Int 86:1072-1074. https://doi.org/10.1038/ki.2014.316

9. Haussler MR, Whitfield GK, Kaneko I, Forster R, Saini R, Hsieh J et al (2012) The role of vitamin D in the FGF23, klotho, and phosphate bone-kidney endocrine axis. Rev Endocr Metab Disord 13: 57-69. https://doi.org/10.1007/s11154-011-9199-8

10. Amanzadeh J, Reilly RF (2006) Hypophosphatemia: an evidencebased approach to its clinical consequences and management. Nat Clin Pract Nephrol 2:136-148. https://doi.org/10.1038/ ncpneph0124

11. Francis F, Hennig S, Korn B, Reinhardt R, De Jong P, Poustka A et al (1995) A gene (PEX) with homologies to endopeptidases is mutated in patients with X-linked hypophosphatemic rickets. Nat Genet 11:130-136. https://doi.org/10.1038/ng1095-130

12. Feng JQ, Clinkenbeard EL, Yuan B, White KE, Drezner MK (2013) Osteocyte regulation of phosphate homeostasis and bone mineralization underlies the pathophysiology of the heritable disorders of rickets and osteomalacia. Bone 54:213-221. https://doi.org/ 10.1016/j.bone.2013.01.046

13. Beck-Nielsen SS, Mughal Z, Haffner D, Nilsson O, Levtchenko E, Ariceta G, de Lucas Collantes C, Schnabel D, Jandhyala R, Mäkitie $\mathrm{O}$ (2019) FGF23 and its role in X-linked hypophosphatemia-related morbidity. Orphanet J Rare Dis 14:58. https://doi.org/10.1186/ s13023-019-1014-8

14. Prié D, Friedlander G (2010) Genetic disorders of renal phosphate transport. N Engl J Med 362:2399-2409. https://doi.org/10.1056/ NEJMra0904186

15. Biosse Duplan M, Coyac BR, Bardet C, Zadikian C, Rothenbuhler A, Kamenicky P, Briot K, Linglart A, Chaussain C (2017) Phosphate and vitamin D prevent periodontitis in X- linked hypophosphatemia. J Dent Res 96:388-395. https://doi.org/10. $1177 / 0022034516677528$

16. Raimann A, Mindler GT, Kocijan R, Bekes K, Zwerina J, Haeusler G, Ganger R (2020) Multidisciplinary patient care in X-linked hypophosphatemic rickets: one challenge, many perspectives. Wien Med Wochenschr 170:116-123. https://doi.org/10.1007/ s10354-019-00732-2

17. Linglart A, Biosse-Duplan M, Briot K, Chaussain C, Esterle L, Guillaume-Czitrom S, Kamenicky P, Nevoux J, Prié D, Rothenbuhler A, Wicart P, Harvengt P (2014) Therapeutic management of hypophosphatemic rickets from infancy to adulthood. Endocr Connect 3:R13-R30. https://doi.org/10.1530/EC-13-0103

18. Connor J, Olear EA, Insogna KL, Katz L, Baker S, Kaur R, Simpson CA, Sterpka J, Dubrow R, Zhang JH, Carpenter TO (2015) Conventional therapy in adults with X-Linked hypophosphatemia: effects on enthesopathy and dental disease. J Clin Endocrinol Metab 100:3625-3632. https://doi.org/10.1210/ JC.2015-2199

19. Beck-Nielsen SS, Brock-Jacobsen B, Gram J, Brixen K, Jensen TK (2009) Incidence and prevalence of nutritional and hereditary rickets in southern Denmark. Eur J Endocrinol 160:491-497. https:// doi.org/10.1530/EJE-08-0818

20. Endo I, Fukumoto S, Ozono K, Namba N, Inoue D, Okazaki R, Yamauchi M, Sugimoto T, Minagawa M, Michigami T, Nagai M, Matsumoto T (2015) Nationwide survey of fibroblast growth factor 23 (FGF23)-related hypophosphatemic diseases in Japan: prevalence, biochemical data and treatment. Endocr J 62:811-816. https://doi.org/10.1507/endocrj.EJ15-0275
21. Rafaelsen S, Johansson S, Ræder H, Bjerknes R (2016) Hereditary hypophosphatemia in Norway: a retrospective population-based study of genotypes, phenotypes, and treatment complications. Eur J Endocrinol 174:125-136. https://doi.org/10.1530/EJE-15-0515

22. Carpenter TO, Imel EA, Holm IA, Jan de Beur SM, Insogna KL (2011) A clinicians' guide to x-linked hypophosphatemia. J Bone Miner Res 26:1381-1388. https://doi.org/10.1002/jbmr.340

23. Hawley S, Shaw NJ, Delmestri A, Prieto-Alhambra D, Cooper C, Pinedo-Villanueva R, Javaid MK (2020) Prevalence and mortality of individuals with X-linked hypophosphataemia: a United Kingdom real world data analysis. J Clin Endocrinol Metab 105. pii: dgz203.

24. Lo SH, Lachmann R, Williams A, Piglowska N, Lloyd AJ (2020) Exploring the burden of X-linked hypophosphatemia: a European multi-country qualitative study. Qual Life ResMar 29:11-1893. https://doi.org/10.1007/s11136-020-02465-x

25. Ferizovic' N, Marshall J, Williams AE, Mughal MZ, Shaw N, Mak C, Gardiner O, Hossain P, Upadhyaya (2020) Exploring the burden of X-Linked hypophosphataemia: an opportunistic qualitative study of patient statements generated during a technology appraisal. Adv Ther 37:770-784. https://doi.org/10.1007/s12325-019-01193-0

26. Skrinar A, Dvorak-Ewell M, Evins A, Macica C, Linglart A, Imel EA, Theodore-Oklota C, San Martin J (2019) The lifelong impact of $\mathrm{x}$-linked hypophosphatemia: results from a burden of disease survey. J Endocrine Soc 3:1321-1334. https://doi.org/10.1210/js. 2018-00365

27. Haffner D, Emma F, Eastwood DM, Duplan MB, Bacchetta J, Schnabel D, Wicart P, Bockenhauer D, Santos F, Levtchenko E, Harvengt P, Kirchhoff M, di Rocco F, Chaussain C, Brandi ML, Savendahl L, Briot K, Kamenicky P, Rejnmark L, Linglart A (2019) Clinical practice recommendations for the diagnosis and management of X-linked hypophosphataemia. Nat Rev Nephrol 15:435-455. https://doi.org/10.1038/s41581-019-0152-5

28. Beck-Nielsen SS, Brusgaard K, Rasmussen LM, Brixen K, BrockJacobsen B, Poulsen MR, Vestergaard P, Ralston SH, Albagha OME, Poulsen S, Haubek D, Gjørup H, Hintze H, Andersen MG, Heickendorff L, Hjelmborg J, Gram J (2010) Phenotype presentation of hypophosphatemic rickets in adults. Calcif Tissue Int 87: 108-119. https://doi.org/10.1007/s00223-010-9373-0

29. Che H, Roux C, Etcheto A, Rothenbuhler A, Kamenicky P, Linglart A, Briot K (2016) Impaired quality of life in adults with X-linked hypophosphatemia and skeletal symptoms. Eur J Endocrinol 174: 325-333. https://doi.org/10.1530/EJE-15-0661

30. Chesher D, Oddy M, Darbar U, Sayal P, Casey A, Ryan A, Sechi A, Simister C, Waters A, Wedatilake Y, Lachmann RH, Murphy E (2018) Outcome of adult patients with X-linked hypophosphatemia caused by PHEX gene mutation. J Inherit Metab Dis 41:865-876. https://doi.org/10.1007/s10545-018-0147-6

31. Marcucci G, Masi L, Ferrari S, Haffner D, Javaid MK, Kamenicky' $P$ et al (2018) Phosphate wasting disorders in adults. Osteoporos Int 29:2369-2387. https://doi.org/10.1007/s00198-018-4618-2

32. Holm IA, Nelson AE, Robinson BG, Mason RS, Marsh DJ, Cowell CT, Carpenter TO (2001) Mutational analysis and genotypephenotype correlation of the PHEX gene in X- linked hypophosphatemic rickets. J Clin Endocrinol Metab 86:38893899. https://doi.org/10.1210/jcem.86.8.7761

33. Ichikawa S, Traxler EA, Estwick SA, Curry LR, Johnson ML, Sorenson AH, Imel EA, Econs MJ (2008) Mutational survey of the PHEX gene in patients with X- linked hypophosphatemic rickets. Bone 43:663-666. https://doi.org/10.1016/j.bone.2008.06.002

34. Horn A, Wright J, Bockenhauer D, Van't Hoff W, Eastwood DM (2017) The orthopaedic management of lower limb deformity in hypophosphataemic rickets. J Child Orthop 11:298-305. https:// doi.org/10.1302/1863-2548.11.170003

35. Skrinar A, Marshall A, San Martin J, Dvorak-Ewell M. X-linked hypophosphatemia (XLH) impairs skeletal health outcomes and 
physical function in affected adults. Poster presented at the ENDO meeting held in San Diego (USA) on 5-8 March, 2015.

36. Cartwright DW, Masel JP, Latham SC (1981) The lumbar spinal canal in hypophosphataemic vitamin D-resistant rickets. Aust NZ J Med 11:154-157. https://doi.org/10.1111/j.1445-5994.1981. tb04223.x

37. Hardy DC, Murphy WA, Siegel BA, Reid IR, Whyte MP (1989) Xlinked hypophosphatemia in adults: prevalence of skeletal radiographic and scintigraphic features. Radiology 171:403-414. https://doi.org/10.1148/radiology.171.2.2539609

38. Cremonesi I, Nucci C, D’Alessandro G, Alkhamis N, Marchionni S, Piana G (2014) X-linked hypophosphatemic rickets: enamel abnormalities and oral clinical findings. Scanning 36:456-461. https://doi.org/10.1002/sca.21141

39. Alon US, Jarka D, Monachino PJ, Sebestyen VanSickle J, Srivastava T (2017) Cinacalcet as an alternative to phosphate therapy in X-linked hypophosphataemic rickets. Clin Endocrinol 87(1):114-116. https://doi.org/10.1111/cen.13346

40. Anagnostis P, Vamvakidis K, Tournis S (2019) Successful management of tertiary hyperparathyroidism associated with hypophosphataemic rickets in an adult. J Musculoskelet Neuronal Interact 19:370-373

41. Srivastava T, Alon US (2013) Cinacalcet as adjunctive therapy for hereditary 1,25-dihydroxyvitamin D-resistant rickets. J Bone Miner Res 28:992-996. https://doi.org/10.1002/jbmr.1838

42. Yavropoulou MP, Kotsa K, Gotzamani Psarrakou A, Papazisi A, Tranga T, Ventis S, Yovos JG (2010) Cinacalcet in hyperparathyroidism secondary to $\mathrm{X}$-linked hypophosphatemic rickets: case report and brief literature review. Hormones (Athens) 9:274-278. https://doi.org/10.14310/horm.2002.1277

43. Raeder H, Shaw N, Netelenbos C, Bjerknes R (2008) A case of Xlinked hypophosphatemic rickets: complications and the therapeutic use of cinacalcet. Eur J Endocrinol 159(Suppl 1):S101-S105. https://doi.org/10.1530/EJE-08-0383

44. Alon US, Levy-Olomucki R, Moore WV, Stubbs J, Liu S, Quarles LD (2008) Calcimimetics as an adjuvant treatment for familial hypophosphatemic rickets. Clin J Am Soc Nephrol 3:658-664. https://doi.org/10.2215/CJN.04981107

45. McHenry CR, Mostafavi K, Murphy TA (2006) Tertiary hyperparathyroidism attributable to long-term oral phosphate therapy. Endocr Pract 12:294-298. https://doi.org/10.4158/EP.12.3.294

46. Nakamura Y, Takagi M, Takeda R, Miyai K, Hasegawa Y (2017) Hypertension Is a Characteristic Complication of X-linked Hypophosphatemia. Endocr J 64(3):283-289. https://doi.org/10. 1507/endocrj.EJ16-0199

47. Nehgme R, Fahey JT, Smith C, Carpenter TO (1997) Cardiovascular abnormalities in patients with X-linked hypophosphatemia. J Clin Endocrinol Metab 82:2450-2454. https://doi.org/10.1210/jcem.82.8.4181

48. Kaya S, Tsuprun V, Hizli O, Schachern PA, Paparella MM, Cureoglu S (2016) Cochlear changes in serous labyrinthitis associated with silent otitis media: a human temporal bone study. Am J Otolaryngol 37:83-88. https://doi.org/10.1016/j.amjoto.2015.10. 002

49. Coyac BR, Hoac B, Chafey P, Falgayrac G, Slimani L, Rowe PS, Penel G, Linglart A, McKee MD, Chaussain C, Bardet C (2018) Defective mineralization in $\mathrm{X}$-linked hypophosphatemia dental pulp cell cultures. J Dent Res 97:184-191. https://doi.org/10. $1177 / 0022034517728497$

50. Alon US, Monzavi R, Lilien M, Rasoulpour M, Geffner ME, Yadin O (2003) Hypertension in hypophosphatemic rickets-role of secondary hyperparathyroidism. Pediatr Nephrol 18:155-158. https://doi.org/10.1007/s00467-002-1044-6

51. Marcucci G, Brandi ML (2021) Congenital conditions of hypophosphatemia expressed in adults. Calcif Tissue Int 108:91103. https://doi.org/10.1007/s00223-020-00695-2

52. Lecoq AL, Brandi ML, Linglart A, Kamenicky P (2020) Management of X-linked hypophosphatemia in adults. Metabolism 103S:154049. https://doi.org/10.1016/j.metabol. 2019.154049

53. Pettifor JM, Thandrayen K (2012) Hypophosphatemic rickets: unravelling the role of FGF23. Calcif Tissue Int 91:297-306. https://doi.org/10.1007/s00223-012-9651-0

54. Koumakis E, Cormier C, Roux C, Briot K (2021) The causes of hypo- and hyperphosphatemia in humans. Calcif Tissue Int 108: 41-73. https://doi.org/10.1007/s00223-020-00664-9

55. Karaplis AC, Bai X, Falet JP, Macica CM (2012) Mineralizing enthesopathy is a common feature of renal phosphate-wasting disorders attributed to FGF23 and is exacerbated by standard therapy in hyp mice. Endocrinology 153:5906-5917. https://doi.org/10. 1210/en.2012-1551

56. Carpenter TO (2012) The expanding family of hypophosphatemic syndromes. J Bone Miner Metab 30:1-9. https://doi.org/10.1007/ s00774-011-0340-2

57. Huang X, Jiang Y, Xia W (2013) FGF23 and phosphate wasting disorders. Bone Res 1:120-132. https://doi.org/10.4248/ BR201302002

58. Gohil A, Imel EA (2019) FGF23 and associated disorders of phosphate wasting. Pediatr Endocrinol Rev 17:17-34. https://doi.org/10. 17458/per.vol17.2019.gi.fgf23anddisordersphosphate

59. Liamis G, Milionis HJ, Elisaf M (2010) Medication-induced hypophosphatemia: a review. Q J Med 103:449-459. https://doi. org/10.1093/qjmed/hcq039

60. Lambert A, Zhukouskaya V, Rothenbuhler A, Linglart A (2019) Xlinked hypophosphatemia: management and treatment prospects. Joint Bone Spine 86:731-738. https://doi.org/10.1016/j.jbspin. 2019.01.012

61. Theodore-Oklota C, Bonner N, Spencer H, Arbuckle R, Chen C, Skrinar A (2018) Qualitative research to explore the patient experience of X-linked hypophosphatemia and evaluate the suitability of the BPI-SF and WOMAC® as clinical trial end points. Value Health 21:973-983. https://doi.org/10.1016/j.jval.2018.01.013

62. Emma F, Cappa M, Antoniazzi F, Bianchi ML, Chiodini I, Eller Vainicher C, di Iorgi N, Maghnie M, Cassio A, Balsamo A, Baronio F, de Sanctis L, Tessaris D, Baroncelli GI, Mora S, Brandi ML, Weber G, D'Ausilio A, Lanati EP (2019) X-linked hypophosphatemic rickets: an Italian experts' opinion survey. Ital J Pediatr 45:67-31. https://doi.org/10.1186/s13052-019-0654-6

63. Mazzucato M, Visonà Dalla Pozza L, Minichiello C, Manea S, Barbieri S, Toto E, Vianello A, Facchin P (2018) The epidemiology of transition into adulthood of rare diseases patients: results from a population-based registry. Int J Environ Res Public Health 15:2212. https://doi.org/10.3390/ijerph15102212

64. De Castro M, Turner C, Kirmse B (2019) Practical recommendations for the transition to adulthood for the adolescent with a genetic diagnosis. Special emphasis on inborn errors of metabolism. Translational Sci Rare Dis 4:159-168. https://doi.org/10.3233/ TRD-190042

65. Lanzkron S, Sawicki GS, Hassell KL, Konstan MW, Liem RI, McColley SA (2018) Transition to adulthood and adult health care for patients with sickle cell disease or cystic fibrosis: current 
practices and research priorities. J Clin Trans Sci 2:334-342. https://doi.org/10.1017/cts.2018.338

66. Shanbhogue VV, Hansen S, Jorgensen NR, Beck-Nielsen SS (2018) Impact of conventional medical therapy on bone mineral density and bone turnover in adult patients with X-linked hypophosphatemia: A 6-year prospective cohort study. Calcif Tissue Int 102:321-332. https://doi.org/10.1007/s00223-0170363-3

67. Sullivan W, Carpenter T, Glorieux F, Travers R, Insogna K (1992) A prospective trial of phosphate and 1,25-dihydroxyvitamin D3 therapy in symptomatic adults with X-linked hypophosphatemic rickets. J Clin Endocrinol Metab 75:879-885. https://doi.org/10. 1210/jcem.75.3.1517380

68. Insogna KL, Briot K, Imel EA, Kamenický P, Ruppe MD, Portale AA, Weber T, Pitukcheewanont P, Cheong HI, Jan de Beur S, Imanishi Y, Ito N, Lachmann RH, Tanaka H, Perwad F, Zhang L, Chen CY, Theodore-Oklota C, Mealiffe M, San Martin J, Carpenter TO, on behalf of the AXLES 1 Investigators (2018) A randomized, double-blind, placebo-controlled, phase 3 trial evaluating the efficacy of Burosumab, an anti-FGF23 antibody, in adults with Xlinked Hypophosphatemia: week 24 primary analysis. J Bone Miner Res 33:1383-1393. https://doi.org/10.1002/jbmr.3475

69. Insogna KL, Rauch F, Kamenicky P, Ito N, Kubota T, Nakamura A, Zhang L et al (2019) Burosumab improved histomorphometric measures of osteomalacia in adults with X-linked hypophosphatemia: a phase 3, single-arm, international trial. J Bone Miner Res 34:2183-2191. https://doi.org/10.1002/jbmr.3843

70. Portale AA, Carpenter TO, Brandi ML, Briot K, Cheong H, CohenSolal M et al (2019) Continued beneficial effects of burosumab in adults with $\mathrm{x}$-linked hypophosphatemia: results from a 24-week treatment continuation period after a 24-week double-blind placebo-controlled period. Calcif Tissue Int 105:271-284. https:// doi.org/10.1007/s00223-019-00568-3

71. DeLacey S, Liu Z, Broyles A, El-Azab SA, Guandique CF, James BC, Imel EA (2019) Hyperparathyroidism and parathyroidectomy in X-linked hypophosphatemia patients. Bone 127:386-392. https://doi.org/10.1016/j.bone.2019.06.025

72. Lee J, Imel EA (2013) The changing face of hypophosphatemic disorders in the FGF-23 era. Pediatr Endocrinol Rev 10:367-379

73. Bjelakovic G, Gluud LL, Nikolova D, Whitfield K, Wetterslev J, Simonetti RG, Bjelakovic M, Gluud C, Cochrane Metabolic and Endocrine Disorders Group (2014) Vitamin D supplementation for prevention of mortality in adults. Cochrane Database Syst Rev 10: CD007470. https://doi.org/10.1002/14651858.CD007470.pub3

74. Crysvita, Summary of product characteristics. Last updated version, February 2020.

75. Saraff V, Nadar R, Hogler W (2020) New Developments in the Treatment of X-Linked Hypophosphataemia: Implications for Clinical Management. Pediatr Drugs 22:113-121. https://doi.org/ 10.1007/s40272-020-00381-8

76. Tiosano D, Hochberg Z (2009) Hypophosphatemia: the common denominator of all rickets. J Bone Miner Metab 27:392-401. https://doi.org/10.1007/s00774-009-0079-1

Publisher's note Springer Nature remains neutral with regard to jurisdictional claims in published maps and institutional affiliations. 